\title{
CAPACIDAD DE RESPUESTA Y CAPACIDAD DE ABSORCIÓN. ESTUDIO DE EMPRESAS MANUFACTURERAS EN MÉXICO
}

\section{Organizational responsiveness and absorptive capacity. Study of manufacturing companies in Mexico}

1- Nacionalidad: Mexicana, Grado: Doctorado, Especialización: Ciencias económico-administrativas, Adscripción: Universidad Autónoma del Estado de México, Correo electrónico: demuner7@yahoo.com, (D) ORCID: http://orcid.org/00000002-4542-9113

2- Nacionalidad: Mexicana, Grado: Doctorado, Especialización: Ciencias económico-administrativas, Adscripción: Universidad Autónoma del Estado de México, Correo electrónico: obecerrilt@uaemex.mx, (D) ORCID: http://orcid.org/00000002-5685-5636

3- Nacionalidad: Mexicana, Grado: Doctorado, Especialización: Economía industrial y relaciones laborales, Adscripción: Universidad Autónoma de Baja California, Correo electrónico: manuel_ibarra@uabc.edu.mx, (D) ORCID: http://orcid. org/0000-0003-4725-0544 


\section{Resumen}

La capacidad de absorción es el mecanismo para que las empresas adquieran, asimilen y exploten nuevo conocimiento del exterior y respondan asertivamente. La orientación estratégica se percibe cuando ese conocimiento se expande para lograr los resultados deseados. El objetivo de esta investigación es analizar la relación de la capacidad de absorción y la capacidad de respuesta, así como evidenciar la moderación de la orientación estratégica en esta relación. Los resultados muestran que las dimensiones de la capacidad de absorción: adquisición, asimilación y explotación del conocimiento externo así como la orientación estratégica son positivos predictores de la capacidad de respuesta organizacional. Mediante un modelo de regresión jerárquica se identifica que la orientación estratégica ni amplifica ni atenúa la relación entre la capacidad de absorción y la capacidad de respuesta. Se espera que la capacidad de respuesta aumente cuando las organizaciones desarrollen capacidad de absorción y orientación estratégica.

Palabras clave: capacidad de absorción, capacidad de respuesta, estrategia.

\section{Abstract}

Absorptive capacity is the mechanism for companies to acquire, assimilate and exploit new knowledge from abroad and respond assertively. Strategic orientation is perceived when that knowledge is expanded to create the desired results. The purpose is to analyze the relationship between absorptive capacity and organizational responsiveness, as well as to demonstrate the moderation of strategic orientation in this relationship. The results show that the dimensions of the absorption capacity: acquisition, assimilation and exploitation of external knowledge as well as strategic orientation are positive predictors of organizational response capacity. Using a hierarchical regression model, it is identified that the strategic orientation neither amplifies nor attenuates the relationship between absorption capacity and response capacity. The response capacity is expected to increase when organizations develop absorption capacity and strategic orientation.

Keywords: absorptive capacity, responsiveness, strategic orientation. 


\section{Introducción}

$\mathrm{E}$ l estudio explora el uso del conocimiento previamente gestado al interior de las organizaciones y su impacto en la capacidad de respuesta organizacional. Se acude a las bases teóricas de capacidad de absorción aportadas por Cohen y Levinthal cuyo argumento señala que los integrantes de la organización adquieren, asimilan y explotan el nuevo conocimiento disponible en el entorno para aprovechar las oportunidades emergentes en el entorno.

El estudio también se fundamenta en el postulado de Huber (1991) respecto al aprendizaje organizacional para crear empresas que responden a entornos cambiantes; Huber argumenta que existen relaciones entre las diferentes dimensiones de la capacidad de absorción y la capacidad de respuesta de la organización y los efectos moderadores de la orientación estratégica; los constructos que estudia son similares a los estudiados por Cohen y Levinthal: adquisición de conocimiento, distribución de información, interpretación de información y memoria organizativa.

El concepto capacidad de absorción ha sido ampliamente aplicado a partir de los estudios de Cohen y Levinthal (1990); sus bases refieren que la adquisición, asimilación y explotación del conocimiento del entorno, combinado con el previamente adquirido conducen al desarrollo de la innovación. El reto de la empresa es desarrollar capacidad de absorción para responder ante un entorno cambiante y desafiante, donde la estrategia empleada puede direccionar el esfuerzo hacia los resultados deseados.

Esta investigación contribuye a la literatura a profundizar en primer lugar, el estudio de las dimensiones de la capacidad de absorción de manera separada en su relación con la capacidad de respuesta. En segundo lugar, se exploran posibles efectos moderadores de la orientación estratégica sobre la relación de la capacidad de absorción y la capacidad de respuesta de la organización. Esto puede ser de gran ayuda para entender por qué algunas empresas son más reactivas que otras. Con estos pronunciamientos, se pretende colaborar a la evidencia empírica del sector manufacturero, por su importante aportación a la economía nacional. En este sentido el objetivo del trabajo es analizar la relación de la capacidad de absorción y la capacidad de respuesta, así como evidenciar la posible moderación de la orientación estratégica en esta relación.

Con este fin se explora la medida en que el conocimiento previo de las medianas y grandes empresas tiene un impacto en su capacidad para responder al ambiente externo, para ello el trabajo se organiza en primer lugar con la revisión teórica que describe la capacidad de absorción como el principal mecanismo de los integrantes de la organización para adquirir, asimilar y explotar el nuevo conocimiento disponible en el entorno; también aborda, a partir del aprendizaje organizacional, la capacidad de respuesta y desarrolla el concepto orientación estratégica. En segundo lugar se presenta la metodología y los resultados encontrados. Finalmente se alude a las conclusiones del trabajo y a futuras investigaciones.

\section{Revisión teórica}

\subsection{Capacidad de absorción}

Con base en el aprendizaje organizacional, Cohen y Levinthal introdujeron el término capacidad de absorción, la definieron como la capacidad que posee una empresa para reconocer el valor de la nueva información 
externa, adquirirla, asimilarla y aplicarla con fines comerciales (Cohen y Levinthal, 1990:128). Más tarde, estos mismos autores visualizaron la capacidad de absorción como estrategia para predecir con mayor precisión la naturaleza de los futuros avances tecnológicos (Cohen y Levinthal, 1994).

La capacidad de absorción constituye uno de los procesos de aprendizaje fundamentales en una empresa por su iniciativa en el fomento de habilidades para identificar, asimilar y explotar conocimiento del entorno. En consecuencia, desarrollar y mantener la capacidad de absorción resulta crítico para la supervivencia a largo plazo de una organización porque puede reforzar, complementar o reorientar la base de conocimientos de la empresa (Lane, Koka y Pathatk, 2006).

La aportación teórica de Zahra y George (2002) enfatiza la integración y distribución del conocimiento interno como las partes más críticas en el desarrollo de la capacidad de absorción por su intención de crear una capacidad organizativa dinámica, que se desarrolla a través de esfuerzos sistemáticos y persistentes orientados a la consecución o sostenimiento de una ventaja competitiva. Estos autores adicionan un concepto, la transformación, e identifican la capacidad de absorción como una capacidad dinámica incorporada en las rutinas y procesos de la empresa que promueve el cambio y la evolución organizacional; para ello establecen dos categorías: capacidades potenciales que involucran la adquisición y asimilación del conocimiento, y capacidades realizadas que incluyen la transformación y la explotación.

La capacidad de absorción ha alcanzado una rápida difusión y aplicación en los estudios organizacionales, donde la unidad de análisis se ha centrado en la empresa y sus actividades inter organizativas (Lane y Lubatkin, 1998), en sus estructuras organizativas, así como en sus unidades de negocio (Jansen, Van Den Bosch y Volberda, 2005).

Los estudios se han intensificado en la búsqueda de los efectos que la capacidad de absorción tiene sobre: la actividad innovadora de la empresa (Becker y Peters, 2000; Cohen y Levinthal, 1990; Nieto y Quevedo, 2005), el desarrollo de nuevos productos (Stock, Greis y Fischer, 2001), proceso de crecimiento empresarial (Barkema y Nadolska, 2003), éxito en los resultados de los acuerdos de cooperación (Newey y Shulman, 2004), la adopción de nuevas prácticas en la organización (Lenox y King, 2004), capacidades combinatorias, expresadas a través de diferentes mecanismos organizativos (Jansen et al., 2005), entre otros.

El fundamento de tales estudios se remonta a los postulados básicos de la capacidad de absorción representados por: a) el conocimiento previo gestado al interior de la organización que hace que las empresas sean más receptivas a la asimilación y transformación del conocimiento, muchas veces influenciada por las habilidades altamente calificadas, un cierto nivel de educación, capacitación y experiencia con que cuenta su personal (Minbaeva, Pedersen, Björkman et al, 2003). B) Por las dimensiones que la conforman y que representan cómo los integrantes de la organización adquieren, asimilan y explotan el nuevo conocimiento disponible en el entorno en que se desenvuelve la empresa con el fin de aprovechar las oportunidades emergentes, antes que sus rivales puedan reconocerlas (Cohen y Levinthal 1994). Y c) el conocimiento externo enfocado a la búsqueda de innovación.

\subsection{Dimensiones de la capacidad de absorción}

La primera de las dimensiones de la capacidad de absorción se refiere a la adquisición como la capacidad de la empresa para identificar, valorar y seleccionar conocimiento externo crítico para sus operaciones (Lane y Lubatkin, 1998; Zahra y George, 2002). En la adquisición, el aprendizaje exploratorio es utilizado 
para reconocer y entender el nuevo conocimiento externo; representa el "generador" de la inteligencia para la organización, debe obtenerse de diferentes fuentes como proveedores, clientes, competencia, del entorno; no pretende ser de uso exclusivo de un departamento o área, es decir, este conocimiento debe fluir por toda la organización, cuanto más conocimiento se pueda recoger en un período dado, mejor funcionará la adquisición de conocimiento.

La segunda dimensión, la asimilación permite convertir el uso del aprendizaje externo en uno más valioso, relaciona el nuevo conocimiento para ser asimilado con lo que las personas ya saben (Cohen y Levinthal, 1990) y con lo que la organización ha institucionalizado. El conocimiento nuevo que se extrae del exterior, individual y organizacional se socializa, internaliza, combina y externaliza, sin llegar a ser estático, se trata de una espiral de conocimiento que nunca se detiene (Nonaka y Takeuchi, 1995). Mientras mas se comprenda, difunda y comparta el conocimiento, sus flujos serán mas significativos.

Por su parte la explotación es la forma en que la empresa usa e implementa el nuevo conocimiento absorbido con fines comerciales y para crear nuevas capacidades en la empresa (Zahra y George, 2002). En la medida en que se adquiera y asimile el conocimiento externo, mayor será la capacidad de trasladarlo a la consecución de una ventaja competitiva (Camisón y Forés, 2010).

En combinación, la adquisición y la asimilación tienen un impacto en los conocimientos adquiridos, por su parte la explotación tiene un efecto directo positivo en el desempeño de la empresa; de tal forma que aquellas empresas que se centran en la adquisición y asimilación de nuevos conocimientos externos son capaces de renovar continuamente su stock de conocimiento, pero pueden sufrir costos de adquisición sin obtener los beneficios de la explotación (Volberda, Foss y Lyles, 2010).

\subsection{Capacidad de respuesta.}

Un tema esencial en la literatura sobre la adaptación de la organización, ha sido el intento para identificar las fuerzas que promueven y transforman las organizaciones en respuesta a los cambios del medio ambiente (Liao, Welsch y Stoica, 2003). La adaptación de la organización ante cambios en su entorno hace énfasis en el rol proactivo que asumen los directivos en la toma de decisiones, desde el monitoreo e interpretación de los cambios en el ambiente, el análisis de oportunidades y amenazas, hasta el diseño de estrategias para enfrentar los cambios (Weick, 1979).

La capacidad de respuesta al entorno tiene que ver con la acción tomada en base al conocimiento adquirido y a las habilidades desarrolladas que potencian a la organización para comprender y actuar efectivamente; se relaciona con el desempeño, la velocidad y la coordinación con que las acciones son implementadas y periódicamente revisadas (Liao et al., 2003). De esta forma las empresas con rápida respuesta tienden a ser competidores superiores reconocidos por su agilidad, ingenio y por su capacidad de aprender de sus errores (Nonaka y Takeuchi, 1995; Jansen et al., 2005).

Desde el aprendizaje organizacional, la capacidad de absorción integra una serie de rutinas necesarias para identificar y utilizar el conocimiento generado en el exterior. Para ello los integrantes de la organización usan su experiencia, habilidades, capacidades de comunicación, el know how que posee la organización para concretar acciones (Cohen y Levinthal, 1990). De este conocimiento dependerá la asertividad para reconocer nuevo conocimiento del entorno, asimilarlo para explotarlo como respuesta al medio ambiente cambiante y a la solución de los problemas que se presenten 
Ante esta perspectiva, este trabajo busca identificar el efecto que la capacidad de absorción tiene en la capacidad de respuesta de la organización, asumiendo que las organizaciones se enfrentan a una vorágine de cambios ambientales que presentan oportunidades y amenazas que ponen en riesgo su supervivencia (Liao et al., 2003). Se pretende contribuir a la evidencia empírica vinculando la capacidad de absorción de las empresas con su capacidad de respuesta en un acercamiento a la comprensión de por qué algunas empresas son más sensibles que otras. Para tal fin se persigue el cuestionamiento sobre ¿Cuál es la relación entre la capacidad de absorción y la capacidad de respuesta de la organización?

La evidencia muestra que las empresas que han desarrollado capacidad de absorción son mas propensas a adaptarse a los cambios ambientales externos. Cohen y Levinthal (1990:136) señalan que "la capacidad de absorción afecta la formación de expectativas de la empresa, permitiéndole predecir con precisión la naturaleza y el potencial comercial de los avances tecnológicos". Su fundamento es el vínculo entre la formación de expectativas o el nivel de aspiración de una empresa y la sensibilidad para detectar oportunidades emergentes en el medio ambiente.

El desarrollo de la capacidad de absorción permite a la empresa apreciar, comprender y valorar las señales ambientales, de esta forma puede explotar cualquier conocimiento crítico que pueda estar disponible, lo cual hace que mientras posea mayores niveles de capacidad de absorción tenderá a ser más proactiva. Estas empresas están mas atentas a la evolución de los entornos y son capaces de aprovechar las oportunidades presentes en el medio ambiente, por el contrario, las empresas con modesta capacidad de absorción tienden a ser más reactivas (Miles y Snow, 2003) .

Zahra y George (2002) mencionan el riesgo que las empresas enfrentan ante las trampas a que acude la competencia y que mientras mas capacidad de absorción desarrolle la empresa, es probable que sean más hábiles y menos confinadas a las trampas. La asimilación de los conocimientos adquiridos externamente permitirá a una empresa generar nuevas combinaciones y sensibilizarse a soluciones alternativas que pueden ser probadas y disminuir el riesgo de las trampas de la competencia. En este sentido se propone:

H1: La adquisición de conocimiento externo se relaciona positivamente con la capacidad de respuesta de la organización de la empresa.

$$
\mathrm{CR}=\beta 1+\beta 2 \mathrm{AD}
$$

$\mathrm{H} 2$ : La asimilación de conocimiento externo se relaciona positivamente con la capacidad de respuesta de la organización de la empresa.

$$
\mathrm{CR}=\beta 1+\beta 2 \mathrm{AS}
$$

H3: La explotación del conocimiento externo se relaciona positivamente con la capacidad de respuesta de la organización de la empresa.

$$
\mathrm{CR}=\beta 1+\beta 2 \mathrm{EX}
$$

\subsection{Orientación estratégica}

Entre las aportaciones mas relevantes que consideran la relación entre la estrategia de la empresa y su capacidad de absorción, se encuentran los trabajos de Liao et al. (2003) y de Lane et al. (2006); estos autores coinciden que la estrategia de una empresa desempeña un papel importante a la hora de determinar 
qué áreas de conocimiento son valiosas, qué áreas deberían asimilarse y en qué áreas debería aplicarse el nuevo conocimiento. La orientación estratégica se percibe cuando las empresas operan de forma "inteligente", cuando son capaces de integrar eficazmente la percepción, la creación del conocimiento y la toma de decisiones porque lleva a las personas a expandir su capacidad de crear los resultados que realmente desean (Choo, 1996), en ellas, las nuevas formas y patrones de pensamiento son experimentadas y las personas aprenden continua y conjuntamente.

La organización conocedora está bien preparada para sostener su crecimiento y desarrollo en un entorno dinámico; cuando percibe éste y comprende su entorno es capaz de prepararse para la adaptación temprana. Al acudir a las habilidades y la experiencia de sus miembros, puede participar en el aprendizaje continuo y la innovación. Al aplicar reglas y rutinas de decisión aprendidas, está preparada para tomar medidas oportunas e intencionadas (Choo, 1996).

Miles y Snow (2003) dicen que para que tenga éxito la organización y para el adecuado desarrollo de toda estrategia, resulta necesario un proceso de adaptación al ambiente del mercado donde la organización es un sistema completo con interacción dinámica donde se identifican ciertas similitudes que distinguen a las empresas. Estos autores postulan que las empresas que compiten dentro de una industria exhiben patrones de comportamiento identificados en cuatro tipos de estrategia competitiva ideales: prospectores, defensores, analizadores y reactores.

A partir de las aportaciones de Liao et al. (2003), los prospectores continuamente experimentan con nuevos productos, se aventuran en nuevos mercados, están más preocupados por la búsqueda de nuevas oportunidades; ponen mayor énfasis en las capacidades de desarrollo de exploración del entorno externo, evalúan amenazas y oportunidades, lo que les permite tomar la iniciativa en el desarrollo de nuevos productos y aventurarse en nuevos mercados. Los defensores tienen un producto estrecho, estable y de dominio de mercado, rara vez hacen ajustes importantes en su tecnología o estructura. Hacen énfasis en formas más eficientes de producción de un determinado producto o servicio.

De acuerdo a esta tipología, determinadas estrategias favorecen el desarrollo de la capacidad de absorción, las empresas prospectoras desarrollan más la capacidad de adquirir, transformar y explotar el conocimiento (Flor, Oltra y García, 2011). Estas empresas se mantienen alertas para encontrar y explotar nuevos productos y oportunidades de mercado, son líderes en su ramo, su éxito depende de examinar una amplia gama de condiciones ambientales, tendencias y acontecimientos (Miles y Snow, 2003) lo que las lleva a considerar suficiente información para tomar decisiones estratégicas, como respuesta competitiva y nuevos diseños de productos.

Por su parte los defensores pueden hacer hincapié en la difusión de conocimientos intrafirma como una forma para mejorar la eficiencia interna en la organización (Liao et al., 2003) tratan de ofrecer un conjunto estable de productos a un segmento del mercado en el que se especializan, fijan su atención a obtener incrementos en la eficiencia de sus operaciones actuales, mejoran los procesos para reducir los costos de fabricación y raramente buscan nuevas oportunidades (Miles y Snow, 2003). Ante estos pronunciamientos:

H4: La orientación estratégica se relaciona positivamente con la capacidad de respuesta de la organización de la empresa.

$$
\mathrm{CR}=\beta 1+\beta 2 \mathrm{OE}
$$


Adicionalmente se plantea la siguiente pregunta ¿En qué medida la relación de la capacidad de absorción y la capacidad de respuesta de la organización, está siendo moderada por la orientación estratégica de las empresas?

A partir de estas consideraciones, se propone:

H5: La orientación estratégica modera la relación entre la adquisición de conocimiento externo y la capacidad de respuesta de la organización.

$\mathrm{CR}=\beta 1+\beta 2 \mathrm{AD}+\beta 3 \mathrm{OE}+\beta 4 \mathrm{OE}^{*} \mathrm{AD}$

H6: La orientación estratégica modera la relación entre la asimilación de conocimiento externo y la capacidad de respuesta de la organización.

$\mathrm{CR}=\beta 1+\beta 2 \mathrm{AS}+\beta 3 \mathrm{OE}+\beta 4 \mathrm{OE}^{\star} \mathrm{AS}$

H7: La orientación estratégica modera la relación entre la explotación de conocimiento externo y la capacidad de respuesta de la organización.

$\mathrm{CR}=\beta 1+\beta 2 \mathrm{EX}+\beta 3 \mathrm{OE}+\beta 4 \mathrm{OE}^{\star} \mathrm{EX}$

\section{Metodología}

La muestra se determinó en base al estudio de las empresas del Estado de México por su importante participación económica a nivel nacional. Según datos del INEGI (2015), durante 2014, el Estado de México ocupó el tercer lugar en la producción bruta total nacional -valor de todos los bienes y servicios producidos o comercializados por cada unidad económica -. Específicamente, nos enfocamos a empresas del municipio de Toluca, se elige éste, por su mayor participación (20.8\%) a la economía estatal. El otro factor de decisión es la posición que la capacidad de empleo provee al estado, Toluca ocupó el primer lugar con una participación del $10.9 \%$. En cuanto al sector, el de manufactura, es quien lidera a los sectores de comercio y servicios. Toluca cuenta con el $64.6 \%$ de empresas manufacturas de todo el Estado. Aunque solo el 3\% de ellas incluye medianas y grandes empresas, es el grupo que mayormente contribuye a la economía. La muestra final quedó conformada por 112 empresas manufactureras de diferentes ramos; 67 de tamaño grande y 35 de tamaño mediano; la tasa de respuesta corresponde al 30\%.

El cuestionario fue auto administrado durante el segundo semestre de 2017. Se aplicó con carácter anónimo a ejecutivos de primer nivel de la empresa. Se compuso por preguntas cerradas a calificarse con una escala tipo Likert de 5 puntos, de 1 (nunca) a 5 (siempre).

El cuestionario se constituyó en primer lugar, con dos variables demográficas que indican el tamaño de la empresa y la rama específica de manufactura a la que se dedica la empresa. En segundo lugar, integra las tres dimensiones: 1) adquisición, definida como la capacidad de la empresa para identificar, valorar y seleccionar conocimiento externo crítico para sus operaciones (Lane y Lubatkin, 1998; Zahra y George, 2002); 2) asimilación: capacidad de la empresa para analizar, procesar, interpretar, internalizar y clasificar el nuevo conocimiento externo adquirido (Zahra y George, 2002); 3) explotación: capacidad para utilizar el nuevo conocimiento con fines comerciales (Cohen y Levinthal, 1994).

Para cada dimensión, se estudiaron las aportaciones teóricas de Cohen y Levinthal $(1990,1994)$ y Zahra y George (2002) por ser pioneros en el tema, y las evidencias empíricas que presentan Nieto y Quevedo (2005), Flor et al. (2011), Fernhaber y Patel (2012) y Chauvet (2014). Cada dimensión se integró por 8 reactivos. 
Las preguntas para adquisición, se refieren a: cómo la empresa detecta señales del medio ambiente e identifica información nueva que puede llevar al interior; qué tan bien juzga la cantidad de conocimientos e información que adquiere de sus clientes, competencia y avances tecnológicos; qué tanta colaboración busca para nuevos procesos o productos; y sí se vincula con externos. En asimilación, se integran preguntas que se enfocan a: qué tan bien la empresa juzga y hace explícito ese conocimiento nuevo con el resto de la administración; su comparación con la competencia en innovaciones, mejoras, lanzamientos; sí su capacitación y especialización le permite desarrollar su capacidad tecnológica; y sí usa ese conocimiento para introducir novedades al mercado mundial. En explotación, las preguntas se enfocan a: qué tanto la empresa está conformada por puestos directivos; su nivel de coordinación para la comercialización; concentración en mas localidades; y cómo se realiza el desarrollo y lanzamiento de productos.

El método parte de la identificación de la calidad métrica del cuestionario. Para ello se realizó un análisis factorial exploratorio con componentes principales y rotación ortogonal Varimax. Se obtuvo con la medida de suficiencia de muestreo (MSA), un determinante de $8.321 \mathrm{E}-5$ muy cercano a cero que indicó la conveniencia de realizar el análisis factorial. La matriz de correlación anti imagen reveló la conveniencia de eliminar dos ítems debido a su presencia de valor $<0.5$. La medida de adecuación de muestreo KaiserMeyer-Olkin presentó valor MASg 0.777 aceptable para el estudio. La prueba de esfericidad de Bartlett (784.9) con Sig. <.05, demostró que existe evidencia de un análisis factorial adecuado. En el análisis de las comunalidades, se eliminaron dos ítems con cargas $<0.5$. Siguiendo la recomendación de Nunnally y Bernstein (1994:535) se integran los factores con ítems cuyas cargas son superiores a 0.5. eliminando tres ítems: Adq1, Explo20, Explo24, cuyas cargas son menores 0.4 (Hair, Anderson, Tatham et al, 1999).

El análisis de factores principales arrojó una estructura factorial mas clara que explica el $51 \%$ de la varianza explicada, que aunque no es alta, se acepta debido a que los ítems muestran una agrupación conceptual (Heisse, 1974, citado en Morales, 2013). Finalmente de los 24 ítems originales quedan, los factores: adquisición (7), asimilación (7), explotación (5) siendo un total de 19 ítems. Se determinó Alfa de Cronbach de cada dimensión estudiada (adquisición 0.665, asimilación 0.810, explotación 0.533) de acuerdo con Nunnally y Bernstein (1994) la fiabilidad es aceptable. El análisis factorial exploratorio permitió deducir que el cuestionario final cumple con las propiedades psicométricas que requiere la literatura.

La capacidad de respuesta de la organización se definió como el conjunto de acciones que refleja la velocidad y coordinación con las acciones que se implementan y revisan periódicamente. También se refiere a la evaluación sobre seguimiento de los objetivos y de la acciones correctivas posteriores (Kohli y Jaworski, 1990). El cuestionario se conformó con 11 ítems (Nieto y Quevedo, 2005), que refieren a la importancia que da la empresa a la segmentación del mercado para el desarrollo de nuevos productos; versan sobre: si sus esfuerzos de desarrollo de productos cumplen lo que quieren los clientes; reuniones para planear acciones que enfrenten los cambios de su entorno empresarial; correspondencia de lo que venden con lo que el mercado necesita; coordinación de departamentos y acciones en la rapidez de respuesta ante cambios en la competencia; medidas preventivas y correctivas inmediatas a quejas de los clientes; planes de marketing; y precios respecto a competidores. Un análisis factorial de componentes principales con rotación varimax arrojó tres factores, permitiendo la agrupación de los 11 ítems originales, el alfa de Cronbach para la capacidad de respuesta de la organización es 0.761 .

La orientación estratégica se definió como el conjunto de decisiones que se toman para la supervivencia de la empresa con un alto nivel de desempeño (orientación al mercado, tecnología, recursos, clientes) 
(Herat y Mahmood, 2014). Se conformó por 8 preguntas dirigidas a: la calidad del producto; esfuerzos destinados al desarrollo de nuevos productos y mantener los existentes; imagen de marca de la empresa; esfuerzos destinados a reducir los costos; precios; cuota de mercado; economía de escala (Liao et al., 2003). El análisis factorial exploratorio permitió la integración de un solo factor con los ítems originales, el Alpha de Cronbach fue 0.761.

En cuanto a la variable de control, tamaño, se considera uno de los más importantes factores organizativos que afectan el comportamiento de las empresas en respuesta a los cambios en el ambiente de mercado (Liao et al., 2003). Por ejemplo, la teoría de contingencia estructural argumenta que las organizaciones tienden a burocratizarse a medida que aumenta de tamaño, lo que dificulta la capacidad de respuesta de la organización. El tamaño para el sector manufactura se define de acuerdo a la Secretaría de Economía de México (mediana: entre 51 y 250 empleados, grande mas de 251 empleados) (DOF, 2009).

Se realizaron correlaciones para probar las primeras hipótesis (H1, H2, H3, H4). Luego se llevó a cabo un análisis de regresión jerárquica. En primer lugar se incluyeron las variables independientes: adquisición, asimilación, explotación, orientación estratégica y tamaño de la empresa, la variable dependiente es la capacidad de respuesta de la organización. En segundo lugar, se rotaron cada uno de los productos cruzados entre la adquisición, asimilación, explotación con la variable orientación estratégica que supone hace un efecto moderador, la significancia de $\mathrm{B}$ y la significancia de $\Delta \mathrm{R} 2$ indicarían el efecto moderador. El análisis de regresión lineal múltiple se aplica para estimar el modelo en el cual la variable dependiente es la capacidad de respuesta, las variables independientes son adquisición, asimilación y explotación, y la variable moderadora orientación estratégica.

\subsection{Resultados}

De acuerdo a los coeficientes de correlación, la adquisición, asimilación y explotación del conocimiento externo se relacionan positivamente con la capacidad de respuesta de la organización, siendo mas alto el efecto que ejerce la asimilación del conocimiento ( $\mathrm{p}<0.01$; Adquisición: 0.317; asimilación: 0.594; explotación: 0.317). HI, H2 y H3 indican que las dimensiones de la capacidad de absorción de las empresas están positivamente relacionadas con la capacidad de respuesta de la organización. Aunque el valor explicativo para la variable asimilación es mayor que el de adquisición y explotación, las tres dimensiones permiten comprobar las hipótesis propuestas. Estas relaciones sugieren que la capacidad de respuesta de la organización incrementa cuando la adquisición, asimilación y explotación del conocimiento externo de la empresa aumentan.

$\mathrm{H} 4$, refiere que la orientación estratégica se relaciona con la capacidad de respuesta de la organización. El resultado de la regresión $(\mathrm{p}<0.01)$ : 0.758 sugiere que la orientación estratégica contribuye a la explicación de la capacidad de respuesta, por lo tanto se comprueba la hipótesis planteada. Incluso esta correlación se manifiesta más alta que las obtenidas en cada dimensión de la capacidad de absorción.

El cuadro 1, presenta los resultados del modelo de regresión sobre la capacidad de respuesta. La variable de control tamaño de la empresa, no es estadísticamente significativo. Lo que sugiere que el tamaño no hace un impacto importante en la capacidad de respuesta organizacional de las empresas.

El modelo $1(\mathrm{R} \wedge=0,074, \mathrm{p}=0,006)$, el modelo $2(\mathrm{R} \wedge=0,396, \mathrm{p}=0,000$ y el modelo $3(\mathrm{R} \wedge=0,108$, $\mathrm{p}=0,001)$ sugieren que la capacidad de respuesta de la organización incrementa cuando la adquisición, 
asimilación y explotación del conocimiento externo de la empresa aumentan Los coeficientes de regresión estandarizados individuales para las tres variables son estadísticamente significativos, (como también se demostró con las correlaciones), se comprueban las hipótesis propuestas $\mathrm{H} 1, \mathrm{H} 2$ y H3.

$\mathrm{H} 4$, refiere que la orientación estratégica es un predictor de la capacidad de respuesta de la organización. El modelo 4 muestra $\left(\mathrm{R}^{\wedge}=0,571, \mathrm{p}=0,000\right)$, que la orientación estratégica contribuye a la explicación de la capacidad de respuesta. Por lo tanto se comprueba H4.

Para comprobar H5, H6 y H7 que predicen que la orientación estratégica ejerce efectos moderadores; se utilizó la regresión jerárquica. Capacidad de respuesta es la variable dependiente y las variables independientes son: adquisición, asimilación, explotación.

Desafortunadamente y contrariamente a lo que se esperaba, ninguno de los modelos 5, 6 y 7 reflejan una interacción significativa. En otras palabras, no hay evidencia de moderación, de tal forma que las hipótesis H5, H6 y H7 son rechazadas. Por lo tanto, la orientación estratégica ni amplifica ni atenúa la relación entre la capacidad de respuesta de la organización con la adquisición, asimilación y explotación del conocimiento externo. Incluso es interesante que, con respecto a la adquisición y la asimilación, la interacción sea negativa, se podría asumir que cuando más potente se hace la orientación estratégica, menos efecto tiene la adquisición y la asimilación del conocimiento externo para la capacidad de respuesta de la organización, pero el coeficiente no es significativo, así que no hay nada a este respecto de lo que se pueda hacer una inferencia estadística confiable. 
Cuadro 1. Coeficientes de regresión sobre la capacidad de respuesta.

\begin{tabular}{|c|c|c|c|c|c|c|c|c|c|c|c|c|}
\hline 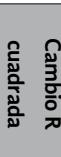 & $\pi$ & 步 & 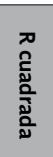 & 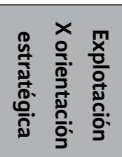 & 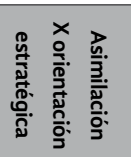 & 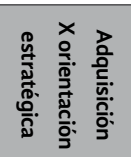 & 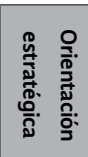 & 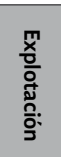 & : & 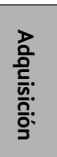 & 売 & 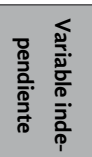 \\
\hline & & $\dot{\dot{g}}$ & 0 & & & & & & & & $\stackrel{\dot{U}}{ }$ & \\
\hline \multirow[t]{2}{*}{$\stackrel{\vdots}{\grave{~}}$} & t: & ¿ & 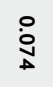 & & & & & & & تِ & & - \\
\hline & & & & & & & & & & 晜 & & -1 \\
\hline \multirow[t]{2}{*}{ 总 } & 总 & 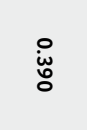 & ڤั & & & & & & : & & & - \\
\hline & & & & & & & & & 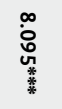 & & & -1 \\
\hline \multirow[t]{2}{*}{ 客 } & 芯 & : & $\stackrel{\circ}{\circ}$ & & & & & 诖 & & & & - \\
\hline & & & & & & & & 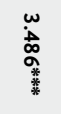 & & & & -1 \\
\hline \multirow[t]{2}{*}{ 离 } & 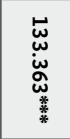 & 喬 & ì & & & & گ̆ & ( & . & & & - \\
\hline & & & & & & & $\begin{array}{l}\vec{E} \\
\vec{w} \\
\vec{w} \\
\vec{t}\end{array}$ & & & & & -1 \\
\hline \multirow[t]{2}{*}{ 总 } & 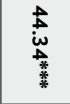 & 总 & 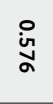 & & & 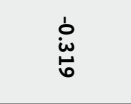 & 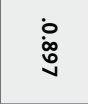 & & & 芯 & & - \\
\hline & & & & & & 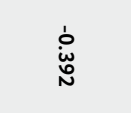 & 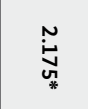 & & & $\stackrel{\$}{\ddagger}$ & & $\dashv$ \\
\hline \multirow[t]{2}{*}{ 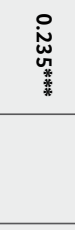 } & 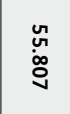 & : & $\stackrel{\stackrel{\circ}{\omega}}{\dot{\omega}^{\prime}}$ & & 㤐 & & $\vec{s}$ & & : & & & - \\
\hline & & & & & 悹 & & 荾 & & 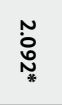 & & & $\dashv$ \\
\hline \multirow[t]{2}{*}{ 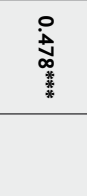 } & 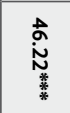 & 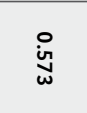 & 总 & 总 & & & ذे & $\dot{:}$ & & & & - \\
\hline & & & & 品 & & & $\tilde{\xi}$ & $\stackrel{\circ}{\circ}$ & & & & -1 \\
\hline
\end{tabular}

$\mathrm{p}<0.05,{ }^{* *} \mathrm{P}<0.01,{ }^{* *} \mathrm{P}<0.001$ - Nota: Coeficientes estandarizados. 


\subsection{Discusión}

Con este trabajo se pretende ahondar en la comprensión del papel que juega la capacidad de absorción de conocimiento externo para desarrollar una capacidad de respuesta ante entornos inciertos. La principal aportación del estudio es que se demuestra cómo las dimensiones que conforman la capacidad de absorción inciden en la capacidad de respuesta de las organizaciones.

Las empresas estudiadas acuden a la adquisición de conocimiento externo, manifestando su interés por detectar señales, tendencias, avances relevantes, que les permiten identificar información que emplean para aumentar su bagaje de conocimiento enfocado a soportar sus respuestas ante cambios en el entorno.

Con la asimilación, este grupo de empresas refleja su capacidad para interpretar y comprender el conocimiento externo; se dirige a implantar en mayor medida procedimientos formales y sistemas para diseminar y absorber el conocimiento con el fin de reducir la incertidumbre del entorno, esto les permite desarrollar habilidades para analizar, procesar y difundir en su interior información útil.

Por su parte, la explotación refiere como estas empresas desarrollan su nivel de coordinación para la comercialización, su alcance va mas allá de lo local y se aventuran al desarrollo y lanzamiento de nuevos productos.

De tal suerte que se comprueba la asociación de la capacidad de absorción como un mecanismo importante que promueve la identificación de fuerzas que transforman a las organizaciones en respuesta a los estímulos del medio ambiente. Con ello, las empresas se mantienen, se posicionan, mejoran su desempeño o alcanzan su visión. Tal como lo sugieren Cohen y Levinthal (1990:136) la capacidad de absorción incide en la formación de expectativas de la empresa, le permite predecir con mayor precisión los avances tecnológicos. En este sentido, las empresas están preparadas para dar rápidas respuestas, se vuelven ágiles e ingeniosas competidoras (Van Den Bosch, Volberda y de Boer, 1999).

Las tres dimensiones parecen ser positivos predictores de la capacidad de respuesta organizacional, sugiriendo que las tres son importantes e interdependientes; son componentes inseparables que permiten a las empresas responder ante cambios en el medio ambiente con fines de innovación o de mejora de desempeño. Estos resultados son coincidentes con los estudios realizados por (Liao et al., 2003), porque aunque las tres dimensiones contribuyen a soportar la capacidad de respuesta, es mas fuerte el esfuerzo que realizan las empresas para asimilar conocimiento y difundirlo en su interior, que adquirirlo y explotarlo.

Adicionalmente, es importante considerar las características internas de las empresas que ayudan a apalancar la capacidad de absorción (Fernhaber y Patel, 2012).

Por su parte la orientación estratégica también contribuye a la explicación de la capacidad de respuesta, como puede verse en la Cuadro 1, es estadísticamente significativa. Similar a los resultados obtenidos por Herat y Rosli (2014) quienes encontraron que las empresas con alto nivel de capacidad de absorción podrían lograr un mayor rendimiento si mantienen un alto nivel de orientación estratégica. Una organización usa información estratégica principalmente para concientizarse de los cambios en el entorno, para crear nuevos conocimientos enfocados al logro de la innovación y para tomar decisiones sobre su curso de acción apropiado; cuando la empresa logra manejar holísticamente estas tres áreas actúa con sabiduría y decisión (Choo, 1996). Según Hakala (2010) para llevar a cabo la actividad empresarial y mantener y mejorar el desempeño organizacional, la orientación estratégica debe visualizarse desde las perspectivas de la orientación al mercado, orientación tecnológica y la orientación al aprendizaje. 
Sin embargo, cuando se analiza si la orientación estratégica modera el efecto en la relación de la capacidad de respuesta y la capacidad de absorción, los resultados no evidencian alguna interacción

\section{Conclusión}

El desarrollo de la capacidad de absorción agiliza la respuesta de las empresas ante la vorágine a que se ven expuestas. Al tratarse de empresas de mayor tamaño, su misma estructura organizativa facilita el acceso a información del exterior, pero esto no garantiza que el conocimiento adquirido se valore con fines de innovación o de mejora de desempeño.

Del desarrollo de la capacidad de absorción depende la asertividad de la empresa para identificar nuevos y valiosos saberes del exterior. Así mismo, la adhesión del nuevo conocimiento a una estrategia hará que la organización esté abierta a la detección de señales que le permitirán encontrar la respuesta adecuada a la solución de problemas o a la búsqueda de los resultados que se plantean, aunque el medio ambiente sea tan cambiante y la competencia amenazante.

Se espera que la capacidad de respuesta de la organización sea respaldada por la capacidad de absorción y que la adopción de una estrategia proactiva pueda conducir a las empresas a su alineación con el entorno externo. Sus directivos deben estar preparados para enfrentarse a condiciones ambientales turbulentas, y prestar más atención a la asignación de recursos para adquirir, asimilar y explotar su conocimiento.

Evidencias empíricas refieren una relación entre la capacidad de absorción y la orientación estratégica desde dos posturas, una es cuando la orientación estratégica puede llevar a las empresas a seleccionar su base de conocimiento e intensidad de esfuerzo de capacidad de absorción de acuerdo con sus necesidades de competencia en el largo plazo; y la segunda, donde la capacidad de absorción fomenta la formación de estrategias.

La principal aportación de este estudio es la evidencia empírica de la relación e importancia que asume la capacidad de absorción como una herramienta potencial de la organización para responder a entornos turbulentos. Así como también se presenta la relación existente entre la orientación estratégica y la capacidad de respuesta, asumiendo que la orientación estratégica de la organización, de acuerdo con (Hakala, 2010) es el mecanismo de adaptación o conjunto de reglas diseñadas y aprendidas para lograr un resultado específico o como comportamientos que ayudan a hacer frente a diferentes entornos porque están diseñados y aprendidos, pueden cambiarse más fácilmente y, por lo tanto, se "usan" gerencialmente para gobernar las actividades de la organización.

Aunque se presenta evidencia de que la orientación estratégica se relaciona con la capacidad de respuesta, este trabajo no encuentra evidencia alguna del efecto moderador en la relación capacidad de absorción y capacidad de respuesta que enfrenta cada organización para responder a entornos turbulentos.

Los resultados de este trabajo podrían justificarse por las similares características de las empresas de la muestra, ya que pertenecen al mismo sector. Queda para futuros trabajos investigar la posible moderación de esta variable entre grupos de empresas de diferentes sectores.

Es importante señalar que una limitación del estudio pudiera ser que la orientación estratégica se ha estudiado de forma general, futuros estudios podrían enfocarse a explorar los efectos mediadores de la orientación estratégica por tipo de estrategia, por ejemplo, la que proponen Miles y Snow. Así como 
investigar si existe un efecto moderador de la orientación estratégica en la relación de la capacidad de absorción y la capacidad de innovación.

Aunque los resultados derivan de una muestra de mas de 100 empresas del sector manufacturero, es importante considerar que se acudió al uso de un diseño de investigación transversal que no permite establecer la causalidad.

\section{Referencias}

Barkema, Harry y Ana Nadolska. 2003. How internationalizing firms develop their absorptive capacity over time: The case of acquisitions. Academy of Management Proceedings.

Becker, Wolfgang y Jüngen Peters. 2000. Technological opportunities, absorptive capacities and innovation. The Eighth International Joseph A. Schumpeter Society Conference. Manchester.

Camisón, César y Beatriz Forés. 2010. Knowledge absorptive capacity. New insights for its conceptualization and measurement. Journal of Business Research, 63: 707-715. DOI: https://doi.org/10.1016/j. jbusres.2009.04.022

Chauvet, Vincent. 2014. Absorptive capacity: Scale development and implications for future research. Management International, 19(1):113-129. DOI: https://doi.org/10.7202/1028493ar

Choo, Chun 1996. The knowing organization: How organizations use information to construct meaning, create knowledge, and make decisions. International journal of Information Management, 16, (5): 329340. DOI: https://doi.org/10.1016/0268-4012(96)00020-5

Cohen, Wesley y Daniel Levinthal. 1990. Absorptive capacity: A new perspective on learning and innovation. Administrative Sciences Quarterly, 35 (1): 128-152. DOI: https://doi.org/10.1016/B978-0-75067223-8.50005-8

Cohen, Wesley y Daniel Levinthal. 1994. Fortune favors the prepared firm. Management Science, 40:227251. DOI: https://doi.org/10.1287/mnsc.40.2.227

Fernhaber, Stephanie y Pankaj Patel. 2012. How do young firms manage product portfolio complexity? The role of absorptive capacity and ambidexterity. Strategic Management Journal, 33 (13): 1516-1539. DOI: https://doi.org/10.1002/smj.1994

Flor, María, María Oltra y Cristina García. 2011. La relación entre la capacidad de absorción del conocimiento externo y la estrategia empresarial: Un análisis exploratorio. Revista Europea de Dirección y Economía de la Empresa, 20, (1): 69-88.

Hair, Joseph, Anderson Rolph, Tatham Ronald y Black William. 1999. Análisis multivariante. Madrid: Prentice Hall.

Hakala, Henri. 2010. Configuring out strategic orientation. Business administration 95, management and organization. Finlandia: University of Vassa.

Herat, H. y Mahmood Rosli. 2014. Strategic orientation and SME performance: moderating effect of absorptive capacity of the firm. Asian Social Science, 10 (13): 95-107. DOI: http://dx.doi.org/10.5539/ass. v10n13p95

Huber, George. 1991. Organizational learning: The contributing processes and the literatures. Organization Science, 2(1): 88-115. DOI: https://doi.org/10.1287/orsc.2.1.88 
DOF. 2009. Diario Oficial de la Federación. Acuerdo por el que se establece la estratificación de las micro, pequeñas y medianas empresas. http://dof.gob.mx/nota_detalle.php?codigo=5096849\&fec ha=30/06/2009 (24 de marzo del 2017).

INEGI. 2015. Censos económicos 2014. http://igecem.edomex.gob.mx/sites/igecem.edomex.gob.mx/ files/files/Archivos\%20PDF/Productos\%20Estadisticos/êndole\%20Social/ISBR/InformacionSBR2015. pdf (15 de enero de 2018).

Jansen, Justin, Frans Van Den Bosch y Henk Volverda. 2005. Managing potential and realized absorptive capacity: How do organizational antecedents matter?. Academy of Management Journal, 48(6): 999-1015. DOI: https://doi.org/10.5465/AMJ.2005.19573106

Kohli, Ajay y Bernard Jaworski. 1990. Market orientation. The construct, research propositions and managerial implications. Journal of Marketing, 54: 1-18. DOI: https://doi.org/10.2307/1251866

Lane, Peter y Michael Lubatkin. 1998. Relative absorptive capacity and inter organizational learning. Strategic Management Journal, 19: 461-477. DOI: https://doi.org/10.1002/(SICI)10970266(199805)19:5<461::AID-SMJ953>3.0.CO;2-L

Lane, Peter, Balaji Koka y Seemantini Pathatk. 2006. The reification of absorptive capacity: A critical review and rejuvenation of the construct. Academic of Management Review, 31: 833-863. DOI: https://doi. org/10.5465/AMR.2006.22527456

Lenox, Michael y Andrew King. 2004. Prospects for developing absorptive capacity through internal information provision. Strategic Management Journal, 25: 331-345. DOI: https://doi.org/10.1002/smj.379 Liao, Jianwen, Harold Welsch y Michael Stoica. 2003. Organizational absorptive capacity and responsiveness: An empirical investigation of growth-oriented SMEs. Entrepreneurship: Theory \& Practice, 28(1): 63-85. DOI: https://doi.org/10.1111/1540-8520.00032

Miles, Raymond y Charles Snow. 2003. Organizational strategy, structure and process. Nueva York: McGraw-Hill.

Minbaeva, D., T. Pedersen, I. Björkman, C. Fey y J. Park. 2003. MNC knowledge transfer, subsidiary absorptive capacity and HRM. Journal of International Business Studies, 34: 586-599. DOI: https://doi. org/10.1057/palgrave.jibs.8400056

Morales, Pedro. 2013. El análisis factorial en la construcción e interpretación de tests, escalas y cuestionarios. Madrid: Universidad Pontificia Comillas.

Newey, Lance y Arthur Shulman. 2004. Systemic absorptive capacity: Creating early-to-market returns through R\&D alliances. R\&D Management, 34: 495-504. DOI: https://doi.org/10.1111/j.14679310.2004.00357.x

Nieto, Mariano y Pilar Quevedo. 2005. Absorptive capacity, technological opportunity, knowledge spillovers, and innovative effort. Technovation, 25: 1141-1157. DOI: https://doi.org/10.1016/j.technovation.2004.05.001

Nonaka, Ikujiro y HirotakaTakeuchi. 1995. The knowledge- creating company: How japanese companies create the dynamics of innovation. Nueva York-Oxford Oxford: University Press.

Nunnally, Jum y Ira Bernstein. 1994. Psychometric theory. Nueva York: McGraw-Hill.

Stock, G., N. Greis y W. Fischer 2001. Absorptive capacity and new product development. Journal of High Technology Management Research, 12(1): 77-92. DOI: https://doi.org/10.1016/S1047-8310(00)00040-7 
Van Den Bosch, Frans, Henrik Volberda y Michiel De Boer. 1999. Coevolution of firm absorptive capacity and knowledge enviroment: Organizational forms and combinative capabilities. Organization Science, 10: 551-568. DOI: https://doi.org/10.1287/orsc.10.5.551

Volberda, Henrik, Nicola Juul y Marjorie Lyles. 2010. Absorbing the concept of absorptive capacity: How to realize its potential in the organization field. Organization Science, 21 (4): 931-951. DOI: https://doi. org/10.1287/orsc. 1090.0503

Weick, Karl. 1979. The social psychology of organizing. Nueva York: McGraw-Hill.

Zahra, Shaker y Gerald George. 2002. Absorptive capacity: A review and reconceptualization. Academy of Management Review, 27: 185-203. 